\title{
Thermal Properties of Buckypaper Enabled Polymer Composites
}

\author{
AYing Zhang 1,a , ZhengHong Li \\ ${ }^{1}$ Harbin University, 150086 Harbin, China \\ ${ }^{2}$ Harbin Institute of Technology, 150001 Harbin, China
}

\begin{abstract}
The heating process of the polymer composite reinforced by buckypaper was simulated with the finite element model. FLUENT was used to analyze how the thickness of buckypaper affect the temperature distribution, the changes of the temperature with time and the heating rate during heating. The maximum and average temperature of the composites decrease when the thickness of the buckypaper increase, whereas the minimum temperature on the samples have the opposite trend. The heating rate of the average temperature of the composites reinforced by the buckypaper with the thickness of $1.2 \mathrm{~mm}$ is faster than those of $0.4 \mathrm{~mm}$ and $0.8 \mathrm{~mm}$. The temperature distribution of the polymer composites reinforced by the buckypaper is more uniform as the thickness of the buckypaper increase.
\end{abstract}

\section{Introduction}

Carbon nanotubes (CNTs) have been comprehensively studied for their excellent electrical, chemical, thermal and mechanical properties [1-4]. However, due to their nanoscale dimension and extra-large surface area, effectively dispersing SWNTs (single wall nanotubes) in a resin matrix for more than $10 \mathrm{wt} . \%$ is exceptionally difficult [5]. Compared with graphite films, buckypaper may exhibit a better thermal conducting performance not only in the plane but also more importantly along the thickness direction. Preparation of the polymer composite material reinforced by the nanopaper is straightforward the dispersion of carbon nanotubes could be more uniform and the structures and functional properties of carbon nanotubes can be further improved. Nanopaper and its reinforced polymer composite materials have extra functions of fire proof, lightening protection and electromagnetic interference shielding. However, previous research on the properties of buckypaper and buckypaper/polymer composites has mostly been concentrated to improve their mechanical properties and conductive properties. In this study, heating model of the polymer composite reinforced by buckypaper is established to predict the thermal property of buckypaper reinforced composite.

\section{Finite element models}

As shown in Figure 1, the heating model of the polymer composites reinforced by the bent buckypaper under different heating conditions were developed by using the finite element program FLUENT. In Figure 1, heating the buckypaper is driven by a power source, and the cube region is the polymer matrix which is heated by the

\footnotetext{
a Corresponding author: zaying@sina.com
}

buckypaper. The temperature of the polymer matrix is increased due to the electro-heating of the buckypaper. The length (L), width (w), and the thickness (T) of the heating model of the polymer composite reinforced by the bent buckypaper are $36 \mathrm{~mm}, 5 \mathrm{~mm}$, and $10 \mathrm{~mm}$ respectively. The thicknesses (d) of the buckypaper are $0.4,0.8$ and $1.2 \mathrm{~mm}$. The bending height (h) and bending period (A) of the bent buckypaper are $6 \mathrm{~mm}$ and $12 \mathrm{~mm}$. The objective of this research is to analyze the effect of the thickness of the buckypaper on the thermal conductivity of the composites using a finite element software FLUENT.

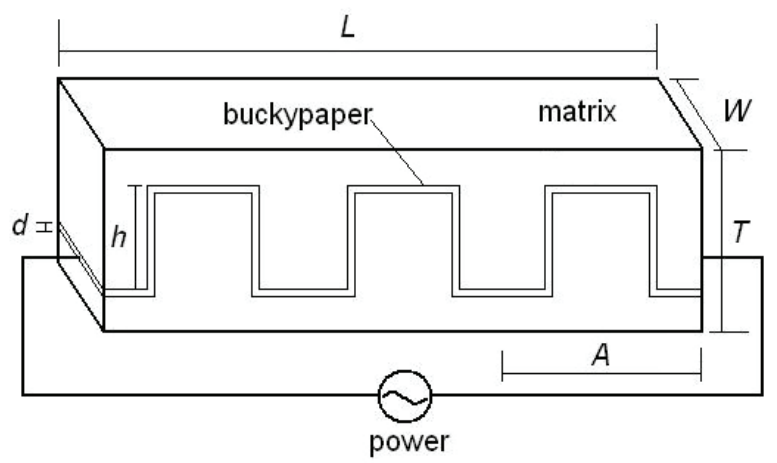

Figure 1. Sketch diagram of heating experimental device

\section{Results and discussion}

As shown in Figure 2, the maximum, minimum and average temperature of the buckypaper/polymer composites reaching a steady state were calculated by the finite element software FLUENT. The maximum, minimum and average temperature of the buckypaper/polymer composites varies linearly with the thickness of buckypaper. 


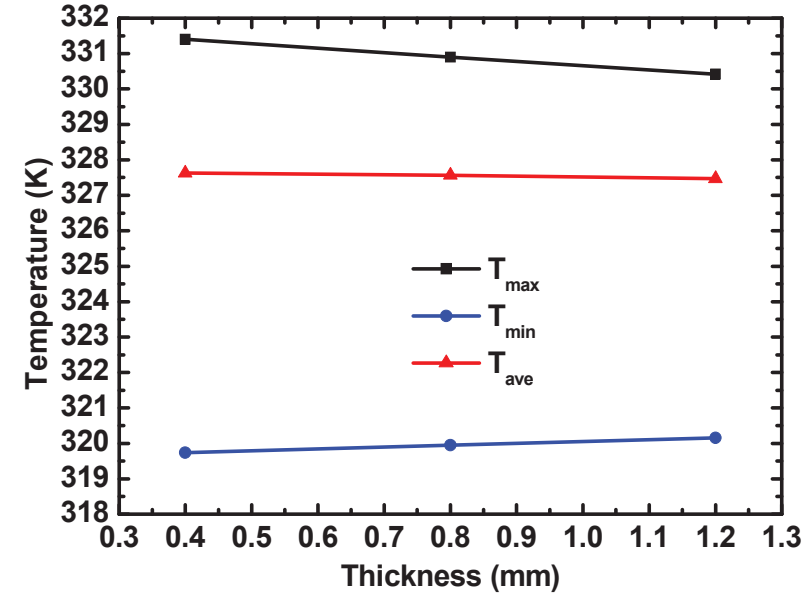

Figure 2. Temperature distribution of the buckypaper/polymer composites along the section $\mathrm{z}=0$

Table 1 shows that the maximum and average temperature decrease as the thickness of buckypaper increase from $0.4 \mathrm{~mm}$ to $1.2 \mathrm{~mm}$. Whereas, the minimum temperature on the samples have the opposite trend.

Table 1. Typical temperature of composites reinforced by buckypaper with different thickness along the section $\mathrm{z}=0$

\begin{tabular}{|c|c|c|c|}
\hline \multirow{2}{*}{$\begin{array}{c}\text { Thickness } \\
\text { /mm }\end{array}$} & \multicolumn{3}{|c|}{ Section of $\mathbf{z}=\mathbf{0}$} \\
\cline { 2 - 4 } & Tmax/K & Tmin/K & Tave/K \\
\hline 0.4 & 331.40 & 319.74 & 327.63 \\
\hline 0.8 & 330.90 & 319.95 & 327.57 \\
\hline 1.2 & 330.42 & 320.15 & 327.47 \\
\hline
\end{tabular}

The heating rate and the time for the buckypaper/polymer composites reaching a steady state were calculated by the finite element software FLUENT. The relationship of the average temperature as a function of time in a transient state along the section $\mathrm{z}=0$ was also analyzed and the results are shown in Figure 3.

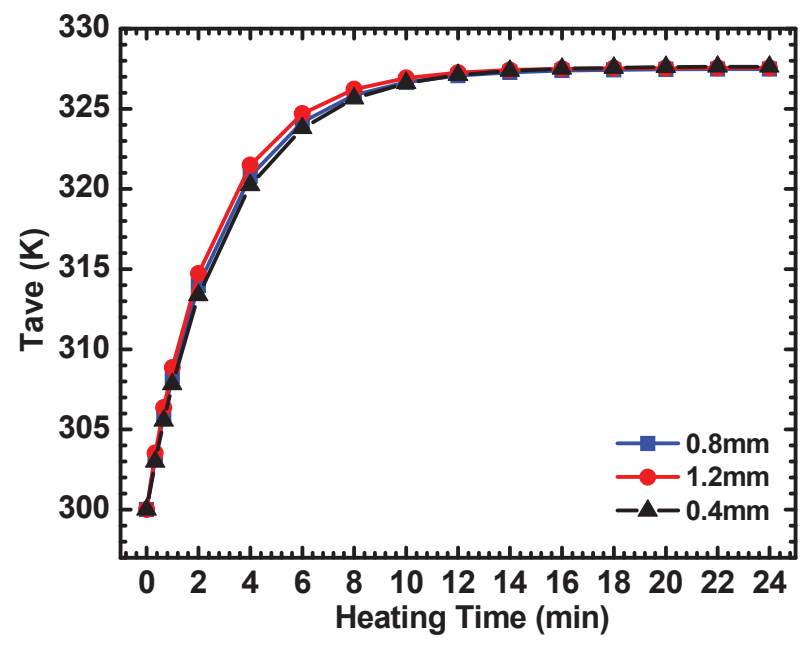

Figure 3. Curve of average temperature of composites reinforced with bending buckypaper of different thickness versus time along the section $\mathrm{z}=0$
Figure 3 shows that both curves of the average temperature exhibit similar trend and there are two distinct stages observed. The first stage shows that the slopes of the curves increase significantly with increasing the heating time. A fast increase of the average temperature occurs during stage I after which the slope is changed, indicating the beginning of the second stage. A progressive but slow increase of the average temperature is observed in the second stage. Figure 3 shows that the heating rate of the average temperature for the polymer composites reinforced by the buckypaper with the thickness of $1.2 \mathrm{~mm}$ is faster than those of $0.4 \mathrm{~mm}$ and $0.8 \mathrm{~mm}$.

Figures 4 and 5 show that the maximum and minimum temperatures of the polymer composites reinforced by the buckypapers of different thickness as a function of time along the section $\mathrm{z}=0$ during heating until reaching the stable state. The maximum temperature of the composites reinforced by the buckypaper with the thickness of $0.4 \mathrm{~mm}$ in a stable state along the section $\mathrm{z}=0$ is higher than that of $1.2 \mathrm{~mm}$, whereas the minimum temperature on the samples have the opposite trend.

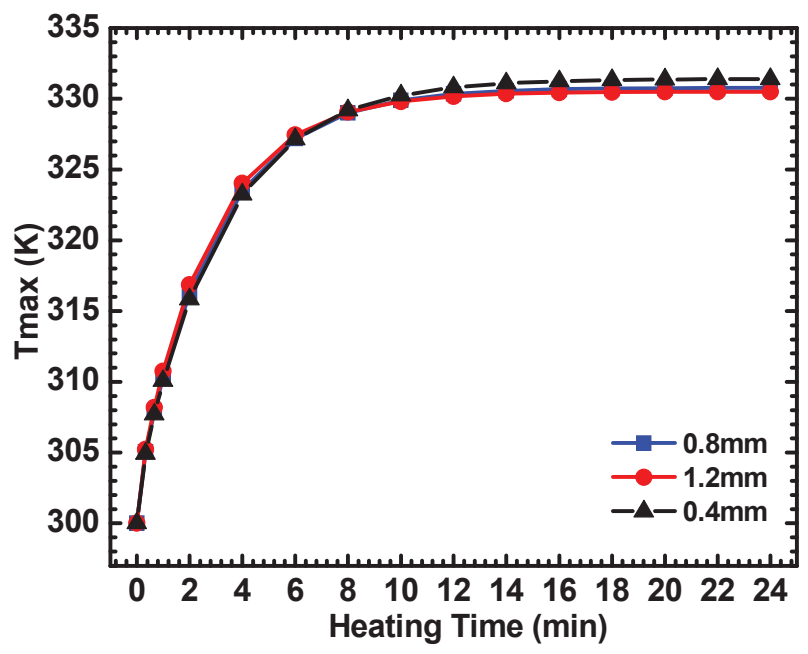

Figure 4. Curve of maximum temperature of composites reinforced with bending buckypaper of different thickness versus time along the section $\mathrm{z}=0$

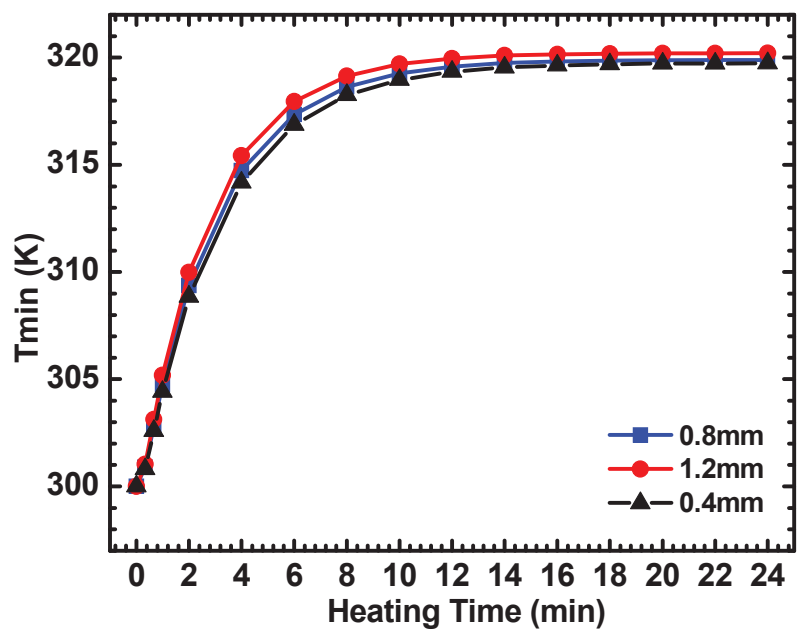

Figure 5. Curve of minimum temperature of composites reinforced with bending buckypaper of different thickness versus time along the section $\mathrm{z}=0$ 
Figure 6 shows the temperature cloudy maps which indicate that the temperature distribution of the polymer composites reinforced by the bent buckypaper along the section $\mathrm{z}=0$ is more uniform as the thickness of the buckypaper increase from $0.4 \mathrm{~mm}$ to $1.2 \mathrm{~mm}$.

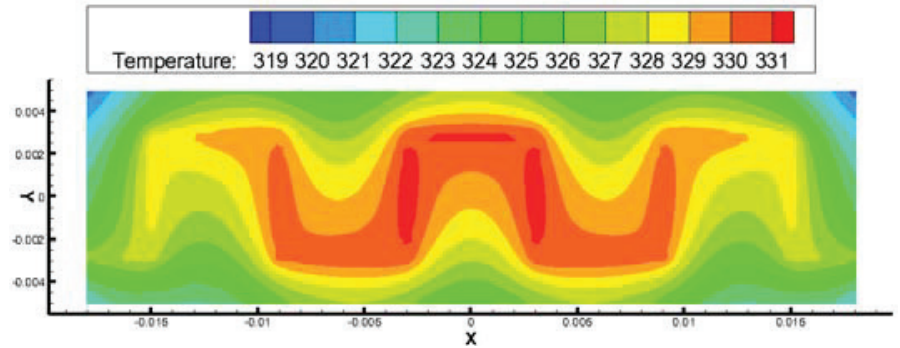

(a) $\mathrm{d}=0.4 \mathrm{~mm}$

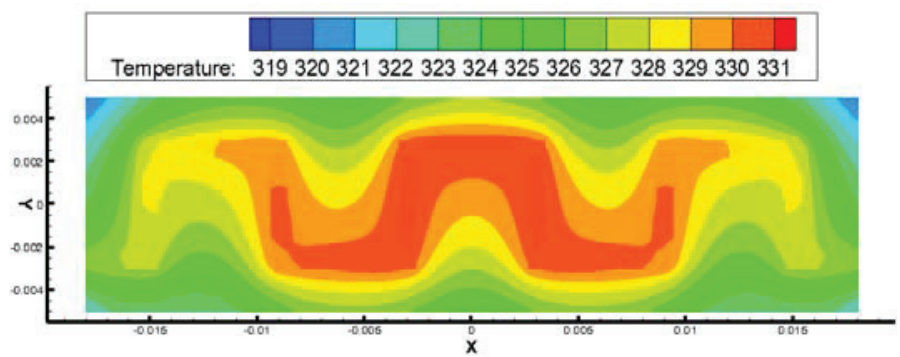

(b) $\mathrm{d}=0.8 \mathrm{~mm}$

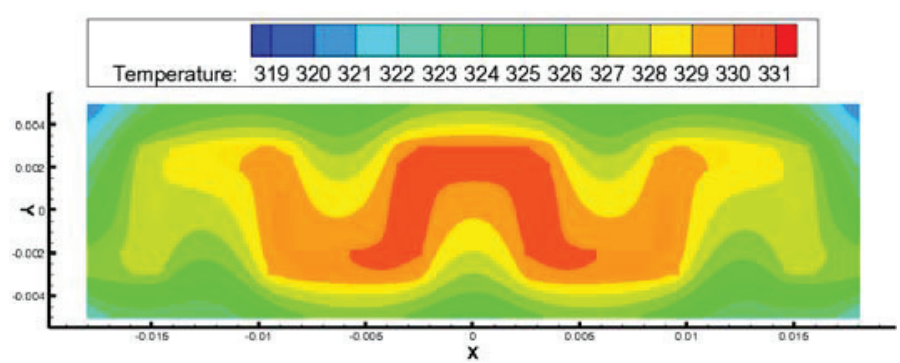

(c) $\mathrm{d}=1.2 \mathrm{~mm}$

Figure 6. Temperature distribution of composites reinforced with bending buckypaper of different thickness along the section $\mathrm{z}=0$

Figure 7 and 8 shows that the temperature distribution of the polymer composites reinforced by the bent buckypaper with the thickness of $0.4 \mathrm{~mm}$ and $0.8 \mathrm{~mm}$ along the section $\mathrm{z}=0$ during heating.

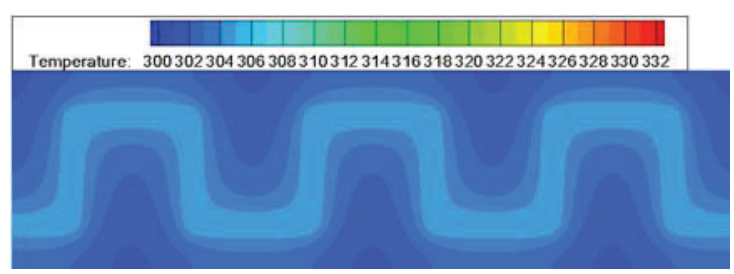

(a) $20 \mathrm{~s}$

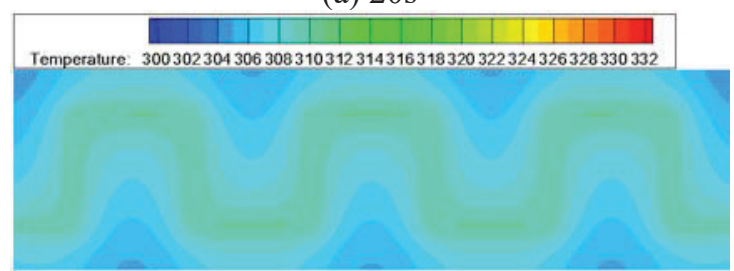

(b) $60 \mathrm{~s}$

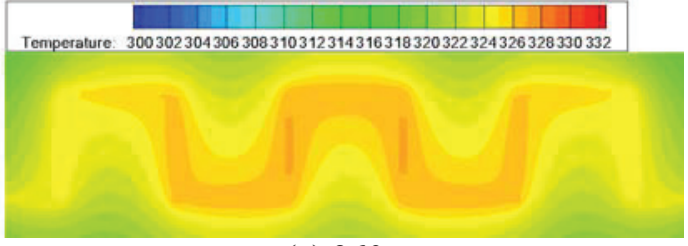

(c) $360 \mathrm{~s}$

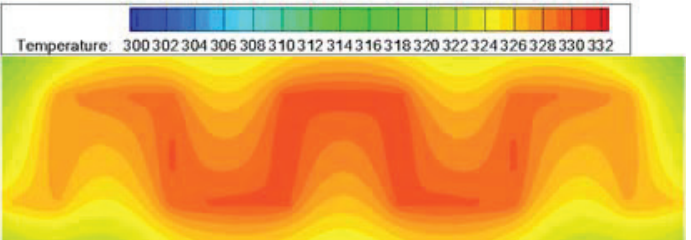

(d) $720 \mathrm{~s}$

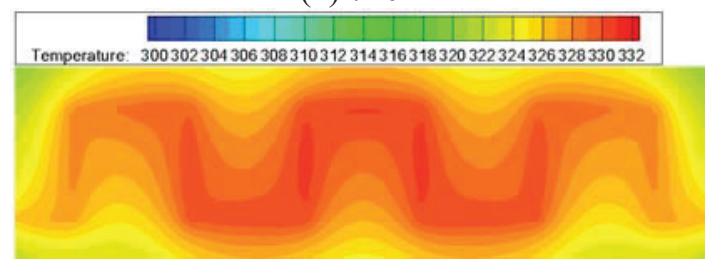

(e) $1080 \mathrm{~s}$

Figure 7. Temperature distribution of composites reinforced with bending buckypaper versus time with the thickness of 0.4 $\mathrm{mm}$ along the section $\mathrm{z}=0$

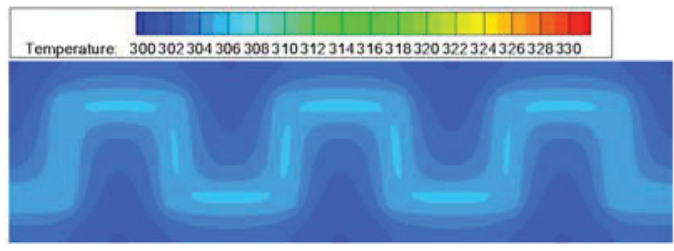

(a) $20 \mathrm{~s}$

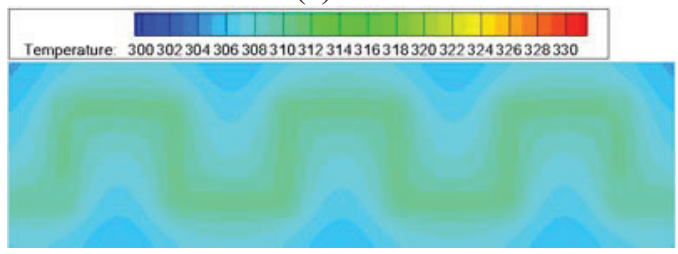

(b) $60 \mathrm{~s}$

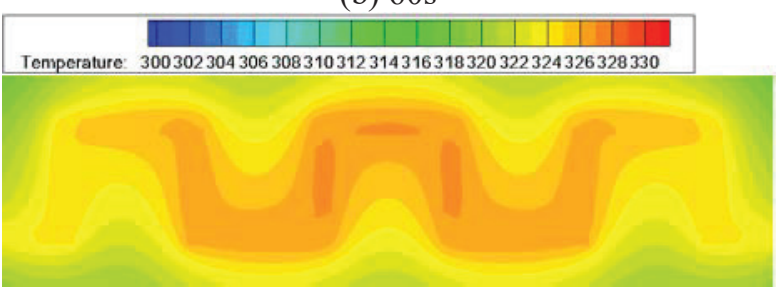

(c) $360 \mathrm{~s}$

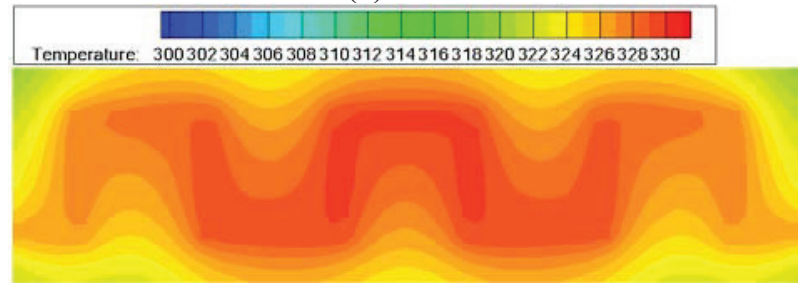

(d) $720 \mathrm{~s}$ 


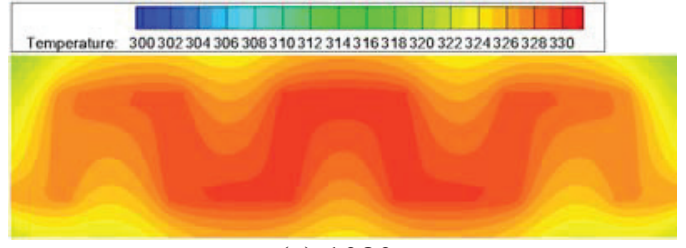

(e) $1080 \mathrm{~s}$

Figure 8. Temperature distribution of composites reinforced with bending buckypaper versus time with the thickness of 0.8 $\mathrm{mm}$ along the section $\mathrm{z}=0$

As shown in Figure 7 and 8, the temperature cloudy maps indicate that the temperature distribution is more uniform as the thickness of buckypaper increase at the same heating time.

Table 2 shows the typical temperature of composites reinforced by the bent buckypaper versus time with the thickness of $0.4 \mathrm{~mm}$ and $0.8 \mathrm{~mm}$ along the section $\mathrm{z}=0$.

Table 2. Typical temperature of composites reinforced by the bent buckypaper versus time with the thickness of $0.4 \mathrm{~mm}$ and $0.8 \mathrm{~mm}$ along the section $\mathrm{z}=0$

\begin{tabular}{|c|c|c|c|c|c|c|}
\hline \multirow{2}{*}{$\underset{/ s}{\text { Time }}$} & \multicolumn{3}{|c|}{$0.4 \mathrm{~mm}$} & \multicolumn{3}{|c|}{$0.8 \mathrm{~mm}$} \\
\hline & $\begin{array}{c}T \max \\
/ \mathbf{K}\end{array}$ & $\begin{array}{c}\text { Tmin } \\
/ \mathbf{K}\end{array}$ & $\begin{array}{c}\text { Tave } \\
/ \mathbf{K}\end{array}$ & $\begin{array}{c}\text { Tmax } \\
/ \mathbf{K}\end{array}$ & $\underset{/ K}{T \min }$ & $\begin{array}{c}\text { Tave } \\
\text { /K }\end{array}$ \\
\hline 0 & 300 & 300 & 300 & 300 & 300 & 300 \\
\hline 20 & 304.93 & 300.80 & 302.98 & 305.04 & 300.90 & 303.24 \\
\hline 40 & 307.70 & 302.59 & 305.54 & 307.89 & 302.84 & 305.93 \\
\hline 60 & 310.07 & 304.42 & 307.83 & 310.35 & 304.77 & 308.31 \\
\hline 120 & 315.83 & 308.86 & 313.37 & 316.28 & 309.38 & 313.99 \\
\hline 240 & 323.23 & 314.18 & 320.23 & 323.54 & 314.74 & 320.80 \\
\hline 360 & 327.14 & 316.87 & 323.79 & 327.19 & 317.35 & 324.17 \\
\hline 480 & 329.19 & 318.26 & 325.64 & 329.00 & 318.64 & 325.84 \\
\hline 600 & 330.25 & 318.97 & 326.60 & 329.90 & 319.28 & 326.67 \\
\hline 720 & 330.81 & 319.35 & 327.10 & 330.35 & 319.59 & 327.08 \\
\hline 840 & 331.10 & 319.54 & 327.36 & 330.57 & 319.75 & 327.28 \\
\hline 960 & 331.25 & 319.64 & 327.49 & 330.68 & 319.82 & 327.38 \\
\hline 1080 & 331.33 & 319.69 & 327.56 & 330.74 & 319.86 & 327.43 \\
\hline 1200 & 331.37 & 319.72 & 327.60 & 330.76 & 319.88 & 327.46 \\
\hline 1320 & 331.39 & 319.73 & 327.62 & 330.78 & 319.89 & 327.47 \\
\hline 1440 & 331.40 & 319.74 & 327.63 & 330.78 & 319.90 & 327.48 \\
\hline
\end{tabular}

Table 2 show that the maximum, minimum and average temperatures of the polymer composites reinforced by the buckypapers of different thickness increase as the thickness of the buckypapers increase. As shown in Table 2, the minimum temperatures of composites increase as the thickness of buckypaper increase at the same heating time, whereas the average temperature on the samples has the opposite trend.

\section{Conclusions}

FLUENT was used to analyze how the thickness of buckypaper affects the temperature distribution, the changes of the temperature with time and the heating rate during heating. The maximum and average temperature of the composites decrease when the thickness of the buckypaper increase, whereas the minimum temperature on the samples have the opposite trend. The heating rate of the average temperature of the composites reinforced by the buckypaper with the thickness of $1.2 \mathrm{~mm}$ is faster than those of $0.4 \mathrm{~mm}$ and $0.8 \mathrm{~mm}$. The temperature distribution of the polymer composites reinforced by the buckypaper is more uniform as the thickness of the buckypaper increase. The minimum temperature of composites increase as the thickness of buckypaper increase at the same heating time during heating, whereas the average temperature on the samples have the opposite trend, which lead to that the temperature distribution is more uniform.

\section{Acknowledgement}

This work was financially supported by Heilongjiang Postdoctoral Scientific Research Developmental Fund (Grant No. LBH-Q16141).

\section{References}

1. R. S. Ruoff, D. C. Lorents. Carbon. 33, 7(1995)

2. J. Hone, J. E. Fischer. Science, 289, 5485(2000)

3. S. Berber, Y. K. Kwon, D. Tomanek. Phys. Rev. Lett., 84, 20(2000)

4. M. A. Osman, D. Srivastava. Nanotechnolgy, 12,1(2001)

5. P. Gonnet, Z. Liang, E. S. Choi, R. S. Kadambala, C. Zhang, J. S. Brooks, B. Wang, L. Kramer. Curr. Appl. Phys., 6, 1(2006) 